\title{
Design of a Novel Mammalian Screening System for the Detection of Bioavailable,
}

\section{Non-cytotoxic Streptogramin Antibiotics}

\author{
DOMINIQUe AUBel ${ }^{a}$, ROWAN MORRIS ${ }^{b}$, BARBARA LENNON $^{c}$, \\ Markus RimanN ${ }^{c}$, Hitto KaUfmanN ${ }^{c}$, Marc Folcher ${ }^{b}$, \\ JAMEs E. BaileY ${ }^{\mathrm{c}}$, Charles J. ThOMPSON ${ }^{\mathrm{b}}$ and MARTIN FussenegGer ${ }^{\mathrm{c}, *}$ \\ a Institut Universitaire de Technologie, IUTA, Département Génie Biologique, \\ 43 Boulevard du 11 Novembre 1918, F-69200 Villeurbanne Cedex, France \\ ${ }^{\mathrm{b}}$ Biozentrum, University of Basel, Department of Microbiology, \\ Klingelbergstrasse 70, CH-4056 Basel, Switzerland \\ ${ }^{c}$ Institute of Biotechnology, Swiss Federal Institute of Technology, ETH Zurich, \\ CH-8093 Zurich, Switzerland
}

(Received for publication July 27, 2000)

\begin{abstract}
Screening and development of new antibiotic activities to counteract the increasing prevalence of multidrug-resistant (MDR) human pathogenic bacteria has once again become a priority in human chemotherapy. Here we describe a novel mammalian cell culture-based screening platform for the detection of streptogramin antibiotics. Quinupristin-dalfopristin (Synercid $^{(\mathbb{B}}$ ), a synthetically modified streptogramin, is presently the sole effective agent in the treatment of some MDR nosocomial infections. A Streptomyces coelicolor transcriptional regulator (Pip) has been adapted to modulate reporter gene expression (SEAP, secreted alkaline phosphatase) in Chinese hamster ovary cells (CHO) in response to streptogramin antibiotics. This CHO cell-based technology was more sensitive in detecting the production of the model streptogramin pristinamycin, from Streptomyces pristinaespiralis, than antibiogram tests using a variety of human pathogenic bacteria as indicator strains. The reporter system was able to detect pristinamycin compound produced by a single $S$. pristinaespiralis colony. The assay was rapid (17 hours) and could be carried out in a high-throughput 96-well plate assay format or a 24-well transwell set-up. This novel mammalian cell-based antibiotic screening concept enables detection of bioavailable and non-cytotoxic representatives of a particular class of antibiotics in a single assay and represents a promising alternative to traditional antibiogram-based screening programs.
\end{abstract}

In recent years, there has been a dramatic worldwide increase in the prevalence of MDR human pathogenic bacteria resulting in an escalation of bacterial disease and related mortality. To counteract this problem, research efforts focusing on the improvement of existing antibiotics by chemical modification have been complemented by the decision of many governments to ban the use of clinically relevant antibiotics and their derivatives, such as the streptogramin virginiamycin, as growth promotants in livestock farming ${ }^{1)}$. Despite the urgent need for anti-infective agents (most antibiotics used today are derivatives of agents which have been in the clinic for more than 30 years), there are few new human-therapeutic antibiotics and efforts to preserve or improve existing compounds cannot keep up with the increasing emergence of new antibiotic-resistant bacterial pathogens ${ }^{2 \sim 8}$ ) (also see U.N. World Health Organization, WHO: The World Health Report (WHO Publications, Geneva, 1996)).

Streptogramins are a unique class of composite antibiotics that are produced by Streptomyces and fungi ${ }^{97}$. These antibiotics consist of two structurally dissimilar compounds, a non-ribosomal cyclic hexadepsipeptide (the type I streptogramin), and a polyunsaturated macrolactone (the type II streptogramin) which are cosynthesized at a

\footnotetext{
* Corresponding: fussenegger@biotech.biol.ethz.ch
} 
constant ratio of $30: 70(\mathrm{w} / \mathrm{w})$ and block the peptidyl transferase catalytic center of the $50 \mathrm{~S}$ ribosomal subunit ${ }^{10)}$. Type I and II components are individually bacteriostatic, but in combination they are bacteriocidal and up to $100-$ fold more active ${ }^{9,11)}$. Synergy between type I and II components results from conformational changes imposed upon the peptidyl transferase center by bound type II component which increases the affinity for the type I streptogramin ${ }^{10,12)}$. Streptogramin antibiotics have desirable pharmacokinetic properties, penetrate most human cells (including macrophages) and resistance rarely develops ${ }^{13,14)}$. Despite these advantages, only two natural streptogramin antibiotics, pristinamycin $\left(\right.$ Pyostacin $^{(B)}$ ) and virginiamycin (Staphylomycin ${ }^{(\mathbb{B})}$ ) are presently available for human therapy. Current research efforts are mainly focused on chemical improvement of existing streptogramins which resulted in development of the first semi-synthetic injectable pristinamycin-derived streptogramin quinupristin-dalfopristin (Synercid ${ }^{\circledR}$ ), a new drug which is licensed for limited use ${ }^{9,11)}$. Quinupristin-dalfopristin has a broad antibiotic spectrum and is efficient against most multidrug-resistant human pathogenic Gram-positive bacteria including vancomycin-resistant species ${ }^{9)}$. Due to its optimized solubility, quinupristin-dalfopristin is currently the prime antibiotic for successful therapy of the difficultto-treat acute endocarditis ${ }^{15)}$.

The low incidence of acquired resistance to streptogramins (a few reported cases in the 50 years of successful streptogramin-based therapy ${ }^{16)}$ ) is a result of their composite nature which requires the presence of both a type I- and a type II-specific resistance determinants ${ }^{5,10,17)}$. Streptogramin-producing Streptomyces such as $S$. pristinaespiralis, a pristinamycin producer, and $S$. virginiae, a virginiamycin producer, typically contain resistance determinants to survive the toxic effects of their own antibiotics they produce ${ }^{18-21)}$. In S. pristinaespiralis, production of pristinamycin is thought to induce expression of the pristinamycin resistance gene $(p t r)^{21)}$. This is mediated by a repressor (Pip; pristinamycin-induced protein) which binds to dyad symmetrical operator sites overlapping the ptr promoter (Pptr) and prevents ptr expression $^{20,22)}$. Pristinamycin binds to Pip and releases it from Pptr which results in derepression of the pristinamycin resistance gene. Based on the pristinamycin-responsive interaction of Pip and its Pptr target sequence, we have recently constructed streptogramin-repressible (PipOFF) as well as streptogramin-inducible (PipON) mammalian gene regulation systems ${ }^{23)}$. The PipOFF system consists of the transactivator PIT (pristinamycin-dependent transactivator), a fusion between Pip and the Herpes simplex VP16 transactivation domain, which binds and activates transcription of its cognate promoter $\left(\mathrm{P}_{\mathrm{PIR}}\right)$ in the absence of pristinamycin. $\mathrm{P}_{\mathrm{PIR}}$ consists of Pptr fused to a minimal eukaryotic promoter. In the PipON configuration, Pip binds to an artificial binding module (PIR3) and blocks transcription of an upstream promoter $\left(\right.$ e.g. $\left.\mathrm{P}_{\mathrm{SV} 40}\right)$ in the absence of pristinamycin. Following interaction with pristinamycin or other streptogramins, Pip is released from the pristinamycin-inducible promoter $\mathrm{P}_{\mathrm{PIR}} \mathrm{ON}\left(\mathrm{P}_{\mathrm{SV} 40}-\mathrm{PIR} 3\right)$ which results in derepression of the promoter ${ }^{23)}$.

Interestingly, the PipOFF as well as the PipON systems were responsive to all commercially available streptogramin antibiotics including Pyostacin ${ }^{\circledR}$, virginiamycin and the forefront drug quinupristin-dalfopristin $\left(\right.$ Synercid $\left.^{\mathbb{R}}\right)$. Here we evaluate the potential of the mammalian PipOFF and PipON gene regulation systems as a screening technology for the detection of bioavailable, non-cytotoxic streptogramin antibiotics. The mammalian cell-based concept is directly compared to traditional antibiogram tests using a variety of human pathogens as indicator bacteria.

\section{Materials and Methods}

Strains, Plasmids and Antibiotics

Bacteria used in this study included: Bacillus subtilis ATCC6631, Listeria monocytogenes, Corynebacterium diphtheriae, Staphylococcus aureus ATCC25932, Staphylococcus aureus (clinical isolate), Streptococcus pyogenes, Streptococcus pneumoniae, Neisseria gonorrhoeae, Neisseria meningitidis, Haemophilus influenzae, and Pseudomonas aeruginosa. The clinical strains (L. monocytogenes, S. aureus, S. pyogenes, $S$. pneumoniae, $N$. gonorrhoeae, $N$. meningitidis, and $H$. influenzae) were isolated from patients hospitalized in Lyon (France), or obtained from the "laboratoire des identifications bactériennes", Institut Pasteur, France ( $C$. diphtheriae CIP100721T). The reference strains $S$. aureus ATCC25923 and the non-pathogenic B. subtilis ATCC6631 were purchased from the American Type Culture Collection (Rockville, MD). B. subtilis, S. aureus and P. aeruginosa were cultivated on Mueller-Hinton agar plates (Sanofi Diagnostics Pasteur, France). The other pathogens were cultivated on Mueller-Hinton agar plates supplemented with 5\% sheep blood (Sanofi Diagnostics Pasteur, France). $H$. influenzae was grown on chocolate-agar plates (BioMérieux, Marcy l'Étoile, France).

Plasmids used in this study include the eukaryotic resistance vectors pSV2neo (Clontech, Palo Alto, $\mathrm{CA}$ ) and pcDNA3.1/Zeo(+) (Invitrogen, Carlsbad, CA), the SEAP 
Table 2. Antibiogram tests.

\begin{tabular}{lccc}
\hline Bacterial Strain & Pristinamycin & Virginiamycin & Supernatant P \\
\hline Bacillus subtilis ATCC6631 & $\mathrm{S}(27)$ & $\mathrm{S}(26)$ & $\mathrm{R}(6)$ \\
Listeria monocytogenes & $\mathrm{S}(34)$ & $\mathrm{S}(32)$ & $\mathrm{R}(6)$ \\
Corynebacterium diphtheriae & $\mathrm{S}(42)$ & $\mathrm{S}(40)$ & $\mathrm{S}(23)$ \\
Staphylococcus aureus ATCC 25932 & $\mathrm{S}(30)$ & $\mathrm{S}(30)$ & $\mathrm{R}(6)$ \\
Staphylococcus aureus & $\mathrm{S}(27)$ & $\mathrm{S}(27)$ & $\mathrm{R}(6)$ \\
Streptococcus pyogenes & $\mathrm{S}(30)$ & $\mathrm{S}(29)$ & $\mathrm{S}(12)$ \\
Streptococcus pneumoniae & $\mathrm{S}(20)$ & $\mathrm{S}(19)$ & $\mathrm{R}(6)$ \\
Neisseria gonorrhoeae & $\mathrm{S}(30)$ & $\mathrm{S}(27)$ & $\mathrm{R}(9)$ \\
Neisseria meningitidis & $\mathrm{S}(28)$ & $\mathrm{S}(25)$ & $\mathrm{R}(7)$ \\
Haemophilus influenzae & $\mathrm{S}(22)$ & $\mathrm{S}(21)$ & $\mathrm{R}(6)$ \\
Pseudomonas aeruginosa & $\mathrm{R}(6)$ & $\mathrm{R}(6)$ & $\mathrm{R}(6)$ \\
\hline
\end{tabular}

Antibiogram discs containing pristinamycin $(15 \mu \mathrm{g})$, virginiamycin $(15 \mu \mathrm{g})$ or pristinamycin-containing Streptomyces pristinaespiralis culture suprnatant $\mathrm{P}$ were tested on $B$. subtilis and a selection of human pathogenic procaryotes. The sensitivity $(\mathrm{S})$ or resistance $(\mathrm{R})$ of the strains to the antibiotics are indicated together with their inhibition diameters $(\mathrm{mm})$. The diameter of the antibiogram dise in $6 \mathrm{~mm}$.

indicator bacteria for the detection of antibiotic activities in metabolic libraries. Antibiotic sensitivity discs loaded with actinomycetes or fungal culture supernatants are placed on a lawn of indicator bacteria and the size of the inhibition diameter measured as an indicator of antibiotic activity. The culture supernatant $\mathrm{P}$ of $S$. pristinaespiralis was tested using the standard indicator strain $B$. subtilis as well as a variety of Gram-positive and Gram-negative human pathogenic bacteria including Listeria monocytogenes, Corynebacterium diphtheriae, Staphylococcus aureus ATCC25932, Staphylococcus aureus (clinical isolate), Streptococcus pyogenes, Streptococcus pneumoniae, Neisseria gonorrhoeae, Neisseria meningitidis, and Haemophilus influenzae which represent the antibiotic spectrum of streptogramins. Pseudomonas aeruginosa was included as a streptogramin-resistant Gram-negative control strain. Most of these pathogenic strains are clinical isolates from infected patients and are sensitive to pristinamycin and virginiamycin in standardized antibiogram tests (see Material and Methods; Table 2). We tested these strains for their susceptibility to pristinamycin concentrations present in $100 \mu \mathrm{l}$ of culture supernatant $\mathrm{P}$ of $S$. pristinaespiralis grown in typical antibiotic production medium for 45 hours. $100 \mu 1$ of supernatant is the maximum amount of liquid which can be added to mammalian cultures grown in multi-well plates.

Results of the antibiogram test using a $S$. pristinaespiralis culture supernatant are shown in Table 2. Only $C$. diphtheriae and $S$. pyogenes were sensitive to the pristinamycin present in $100 \mu \mathrm{l}$ supernatant disc. However, due to safety concerns, the use of highly human pathogenic $C$. diphtheriae and S. pyogenes isolates as indicator strains in high-throughput screening programs obviously would not be advisable. This antibiogram test exemplifies two major limitations of the classical antibiotic screening technology. First, the tester strains have to be susceptible to potential target antibiotics and, second, high antibiotic concentrations are required for reliable detection of antibiotic activities in culture supernatants. The sensitivity of screening assays is of prime importance since antibiotic production of uncharacterized Streptomyces isolates in standard production medium may be suboptimal and/or the 
Fig. 1. Specificity of the CHO-S cell line for streptogramin antibiotics.

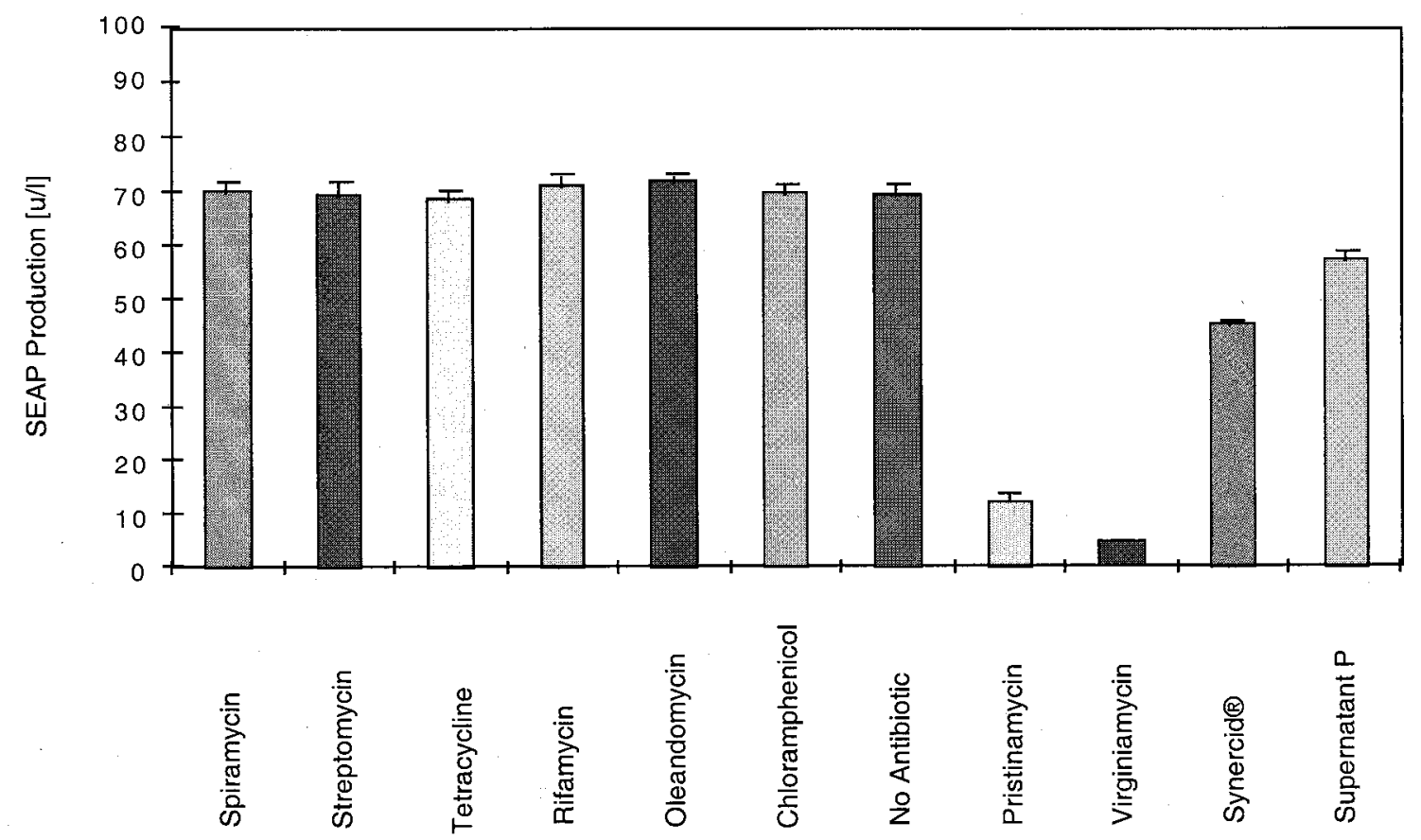

Various antibiotics were added to CHO-S cell cultures at a concentration of $2 \mu \mathrm{g} / \mathrm{ml}$ and SEAP expression was measured after 48 hours. Only streptogramin antibiotics (pristinamycin (Pyostacin ${ }^{B}$ ), virginiamycin, quinupristindalfopristin $\left(\right.$ Synercid $\left.{ }^{(B)}\right)$ ) were able to modulate SEAP expression. In addition, the regulation performance of $100 \mu 1$ culture supernatants derived from S. pristinaespiralis (see Material and Methods) was assessed using CHO-S cells.

antibiotic may be unstable in aqueous solutions (generally the case for streptogramins). In the configuration presented here, the standard antibiogram test using B. subtilis as indicator bacteria would have failed to detect $S$. pristinaespiralis as a streptogramin producer.

\section{CHO-S as Screening Platform for the Detection of Streptogramins}

CHO-S was constructed to be a highly sensitive streptogramin sensor. Instead of using a physiological readout (inhibition of bacterial growth) as in the antibiogram test, the CHO-S-based detection technology relies on the direct interaction of the antibiotic and the Streptomyces streptogramin sensor Pip which is linked to a transcription readout within a mammalian cell. $\mathrm{CHO}-\mathrm{S}$ was able to reliably identify this type of streptogramin in the supernatant as seen by significant reduction in SEAP expression compared to full expression in the absence of any antibiotic (Figure 1). Based on the correlation between the streptogramin concentration and the SEAP readout of CHO-S shown in Table 1, the pristinamycin concentration of the supernatant $\mathrm{P}$ was estimated to be between $0.5 \mu \mathrm{g} / \mathrm{ml}$ and $1 \mu \mathrm{g} / \mathrm{ml}$.

In preliminary experiments using the $\mathrm{CHO}-\mathrm{S} / \mathrm{CHO}-\mathrm{C}$ screening technology as well as $C$. diphtheriae-based antibiograms, 50 culture supernatants of uncharacterized Streptomyces isolates were assayed for streptogramin activities. Two supernatants (\#48 and \#49) showed significant inhibition diameters of $17 \mathrm{~mm}$ (supernatant \#48) and $12 \mathrm{~mm}$ (supernatant \#49) on a C. diphtheriae lawn. These findings correlated with significantly decreased SEAP expression in the CHO-S/CHO-C system (CHO-S:

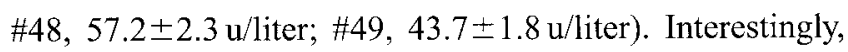
whereas SEAP expression levels of CHO-C remained unchanged following addition of supernatant \#48, supernatant $\# 49$ reduced SEAP expression of CHO-C by about two thirds. This suggested the presence of a cytotoxic compound; such effects may prevent use in antibiotic chemotherapy. Efforts are currently undertaken to isolate the antibiotic produced by Streptomyces strain $\# 48$. 
Fig. 2. Regulation performance of $\mathrm{CHO}$ cells transfected with the PipON system (Pip (pMF150) and $\mathrm{P}_{\mathrm{PIR}}$ ON-SEAP (pMF208)).

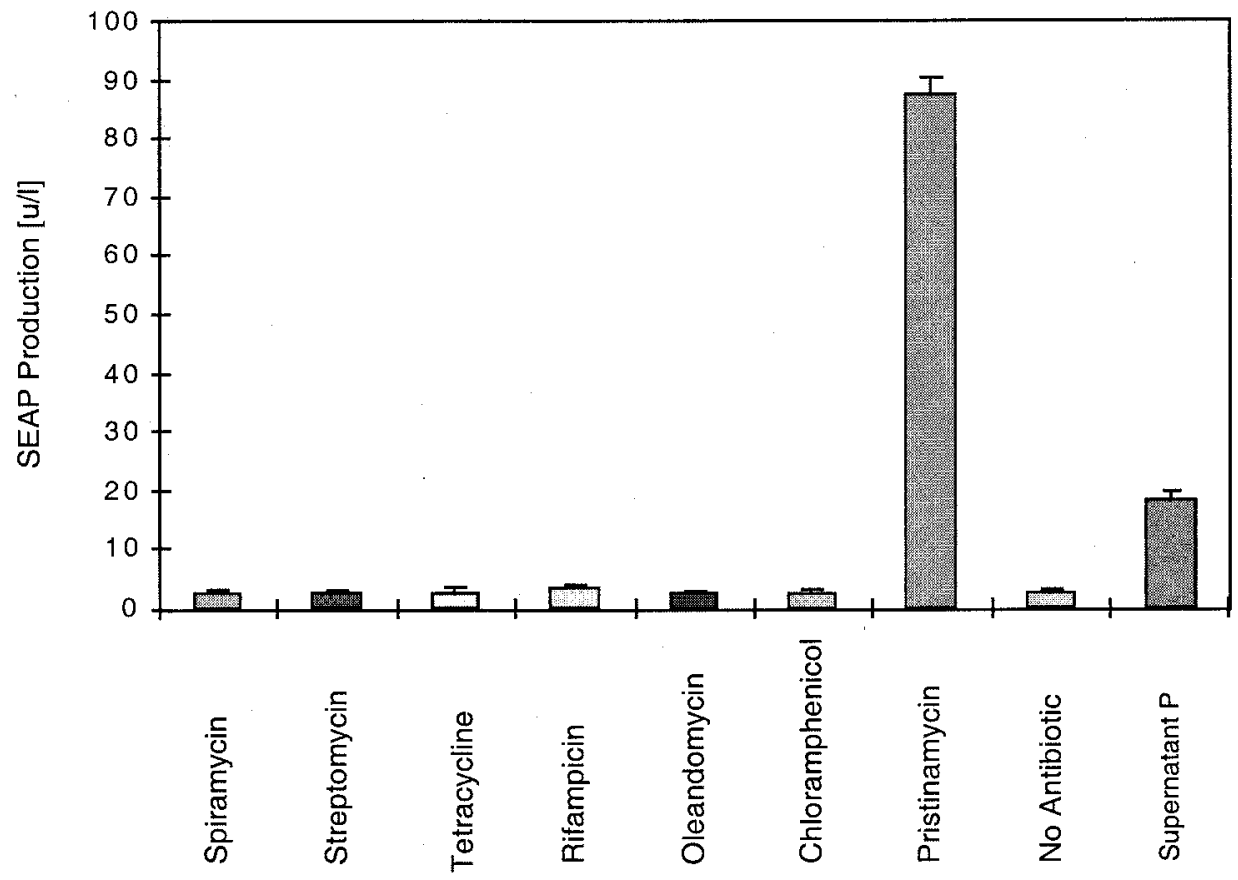

All antibiotics $(2 \mu \mathrm{g} / \mathrm{ml})$ were added to CHO cells harboring the PipON system and SEAP expression was measured 48 hours post transfection. Also, $100 \mu \mathrm{l}$ of $S$. pristinaespiralis culture supernatants were assessed for their SEAP-inducing potential.

Table 3. Correlation between streptogramin concentration and SEAP expression of $\mathrm{CHO}$ cells transfected with the PipON system ((pMF150 (Pip) and pMF208 ( $\left.\mathrm{P}_{\mathrm{PIR}} \mathrm{ON}-\mathrm{SEAP}\right)$ ).

\begin{tabular}{cc}
\hline $\begin{array}{c}\text { Pristinamycin concentration } \\
(\mathrm{ng} / \mathrm{ml})\end{array}$ & $\begin{array}{c}\text { SEAP expression } \\
(\mathrm{u} / \mathrm{liter})\end{array}$ \\
\hline 0 & $2.5 \pm 0.3$ \\
0.5 & $2.5 \pm 0.2$ \\
1 & $2.5 \pm 0.4$ \\
10 & $3.1 \pm 0.1$ \\
50 & $16.1 \pm 0.1$ \\
100 & $25.5 \pm 1.1$ \\
250 & $57.8 \pm 0.3$ \\
500 & $86.0 \pm 2.8$ \\
1,000 & $92.7 \pm 2.0$ \\
2,000 & $87.4 \pm 2.6$ \\
5,000 & $84.7 \pm 3.1$ \\
\hline
\end{tabular}

Transfected $\mathrm{CHO}$ cells were incubated with varying concentrations $(\mathrm{ng} / \mathrm{ml})$ of pristinamycin and the SEAP expression was assessed (units/liter) 48 hours following transfection.
The Use of the PipON System Provides an Internal Control for Toxicity

The initial CHO-S/CHO-C streptogramin detection technology was based on the PipOFF expression system which indicates the presence of putative streptogramin antibiotics by a decrease in SEAP readout. To preclude that the reduction in SEAP expression results from a cytotoxic compound or one which inhibits metabolism, this streptogramin screening set-up requires a control test using the constitutively SEAP-producing $\mathrm{CHO}-\mathrm{C}$ cell line. The use of the PipON expression technology eliminates the need for a cytotoxicity test since interaction of a streptogramin with the Pip molecule will lead to induction of SEAP expression. We have tested the collection of nonstreptogramin antibiotics described above (Figure 1) as well as supernatants $\mathrm{P}, \# 48$ and $\# 49$ in $\mathrm{CHO}-\mathrm{K} 1$ cells which have been cotransfected with the Pip-encoding plasmid pMF150 and the $\mathrm{P}_{\mathrm{PIR}} \mathrm{ON}$-driven SEAP expression construct (pMF208). Figure 2 shows the induction profile of these antibiotics and supernatants compared to a response elicited by $2 \mu \mathrm{g} / \mathrm{ml}$ pristinamycin. The streptogramin concentration 
Fig. 3. Micro assay for streptogramin biosynthesis in a single bacterial colony.
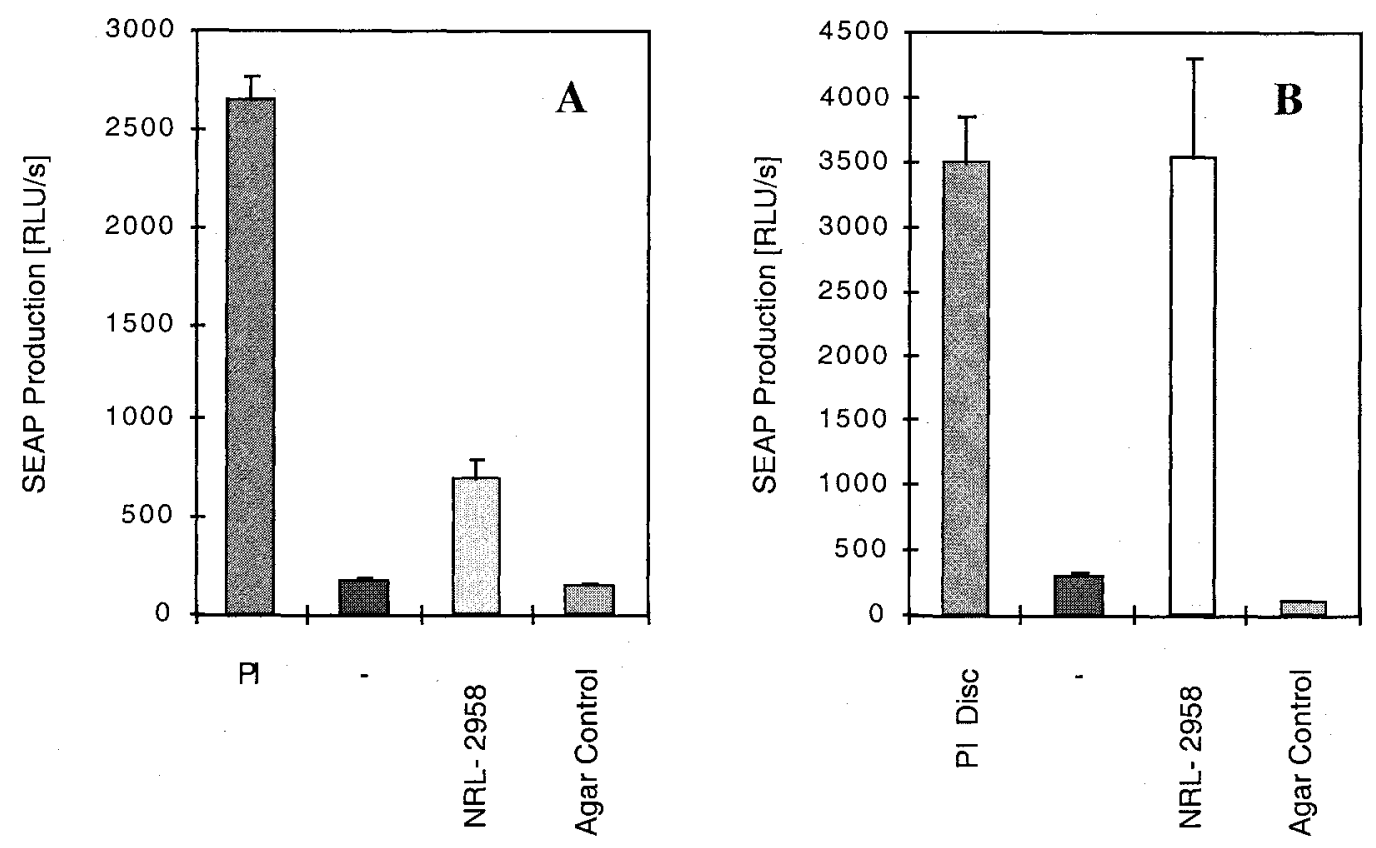

(A) Agar plugs either containing a single S. pristinaespiralis NRRL-2958 colony or no bacteria (agar control) were removed from agar plates using a $1 \mathrm{~mm}$ Pasteur pipette and added to a 96-well plate harboring CHO-K1 cells transfected with the PipON expression system.

(B) A single $S$. pristinaespiralis NRRL-2958 colony was grown on agar in a $6.5 \mathrm{~mm}$ transwell chamber which was transferred to a 24-well plate harboring CHO-K1 cells transfected with the PipON expression system. SEAP expression (relative light units per second, [RLU/s]) was assessed 17 hours after transfection using a chemiluminescence-based phosphatase assay. The values indicated are the result of three independent experiments using three different agar plugs and colonies. The one-colony assay was compared to a control set-up using either no antibiotic or $2 \mu \mathrm{g} / \mathrm{ml}$ pristinamycin.

in supernatant $\mathrm{P}$ (between $0.5 \mu \mathrm{g} / \mathrm{ml}$ and $1 \mu \mathrm{g} / \mathrm{ml}$ ) was comparable to the one determined using CHO-S-based detection technology (PipOFF) as estimated by comparison with the PipON-specific streptogramin-SEAP correlation (Table 3). Whereas supernatant \#48 shows significant induction of SEAP expression $(17.5 \pm 0.87 \mathrm{u} /$ liter $)$, the expression levels of this reporter gene are below the basal PipON expression levels which confirms the cytotoxic characteristic of this supernatant $(1.9 \pm 0.2 \mathrm{u} /$ liter $)$.

The Basis for a Straightforward High-throughput Streptogramin Detection Platform: Detection of Pristinamycin Produced by a Single S. pristinaespiralis Colony

All antibiotic detection systems developed so far require isolation and preliminary characterization of the candidate antimicrobial producer as well as production of antibiotic test batches for different screening assays. These requirements are time-consuming and costly, a major reason why key pharma companies often prefer channeling their resources into rational drug design than in empirical screening programs ${ }^{8}$. The PipON and PipOFF systems had an enhanced sensitivity for the detection of streptogramin antibiotics, a class of compounds having desirable pharmaceutical properties. The assay was therefore adapted to a high-throughput screening platform able to detect putative streptogramins produced by a single Streptomyces colony grown on agar plates. This most advanced variant of the streptogramin screening technology is based on the PipON system. CHO-K1 cells cotransfected with a Pip expression construct (pMF150) and a Pip-responsive SEAP reporter construct (pMF208; $\mathrm{P}_{\mathrm{PIR}} \mathrm{ON}_{\mathrm{SEAP}}{ }^{23)}$ ) were seeded into 96-well plates. An agar plug removed from a plate supporting growth of a $S$. pristinaespiralis colony, grown for 40 hours, was sampled using the tip of a Pasteur pipette ( $1 \mathrm{~mm}$ diameter). Figure $3 \mathrm{~A}$ shows the chemiluminescencebased SEAP readout 17 hours after addition of $S$. 
pristinaespiralis colonies or pure pristinamycin. This prototype one-colony set-up proved to be sensitive enough to detect pristinamycin produced by only a single colony; SEAP expression was significantly induced compared to controls containing Streptomyces-free agar plugs (Figure 3A). The one-colony assay only requires handling of liquids and pipetable agar plugs which renders this screening format compatible with common robotic high-throughput systems. In addition to the 96-well plate screening format we have established a transwell high throughput system which involves growing the $S$. pristinaespiralis colony in an agar-containing transwell chamber. Following 40 hours of incubation, this transwell chamber was placed into a 24 well containing CHO-K1 expressing the PipON system. Pristinamycin production from the $S$. pristinaespiralis colony diffused through the base of the transwell chamber and induced SEAP expression which was measured after 17 hours. Compared to the negative controls (no antibiotic; agar only) the SEAP expression by the S. pristinaespiralis colony was significantly induced and similar to the PI antibiogram disc (Figure 3B). Unlike the 96-well plate format, there is no direct contact between potential streptogramin producers and the screening cells in the transwell set-up which minimizes interference in the screening assay.

\section{Discussion}

The incidence of infections caused by multidrug-resistant human pathogens is dramatically increasing. Some strains of disease-causing bacteria are currently untreatable and produce fatal infections because of their resistance to available antibacterial agents ${ }^{29)}$. Problematic pathogens include strains of Streptococcus pneumoniae resistant to $\beta$ lactams and erythromycin A, Sreptococcus pyogenes resistant to erythromycin $\mathrm{A}$ and multi-resistant strains of Listeria, Clostridium, Corynebacterium and Mycobacterium $^{2}$. Multidrug-resistant human pathogens are responsible for a wide spectrum of diseases including foodborne infections caused by Clostridium (e.g. C. perfringens and C. botulinum) and Listeria monocytogenes, hospital-acquired infections, particularly following invasive surgery, caused by enterococci (e.g. E. faecium) and staphylococci (e.g. S. aureus), and epidemics resulting from common infections by streptococci (e.g. S. pneumoniae) and Corynebacterium diphtheriae ${ }^{2}$. In addition, increasing evidence correlates latent bacterial infections with other pathologies for morbidity and mortality in industrial countries. These include many cardiovascular diseases (particularly coronary heart disease and myocardial infarction), atherosclerosis caused by Chlamydia infection $^{30,31)}$, gastrointestinal ulcers and other neoplastic disorders caused by Helicobacter pylori ${ }^{32)}$ and certain types of arthritis resulting from persistent infections by Neisseria spp. ${ }^{33,34)}$.

There is an urgent need for new antibacterial agents that are able to overcome drug resistance mechanisms ${ }^{3,4,8)}$ (also see U.N. World Health Organization, WHO: The World Health Report (WHO Publications, Geneva, 1996)). In recent years, increasing efforts have focused on improving effective antibiotics by chemical modification ${ }^{9,35,36)}$, combinatorial chemistry ${ }^{37)}$, or genetic engineering of antibiotic biosynthesis pathways ${ }^{38,39)}$. Although some of these strategies have generated valuable therapeutics, the rational design of novel antibiotics is tedious, timeconsuming and unpredictable regarding their therapeutic potential, bioavailability and cytotoxicity characteristics.

Isolation of novel antibiotic molecules from metabolic or compound libraries may represent a powerful alternative to the construction of semi-synthetic derivatives of therapeutically proven antibiotics. Detection of novel antibiotic activities has been limited in the past 30 years by a deficit in sophisticated and sensitive screening technologies ${ }^{8)}$. Two major factors may limit the successful detection of antibiotic activities in antibiogram tests: (i) The concentration of putative antibiotics in metabolic libraries is not sufficiently high to induce a significant inhibition diameter, or (ii) the indicator strain is resistant to the screening compound. Resistance of indicator bacteria to candidate antibiotics is an important parameter since their susceptibility varies greatly between different species and even between different isolates of the same species (see Table 2 for $S$. aureus as an example). For safety reasons, the use of non-pathogenic $B$. subtilis as Gram-positive indicator strain has become standard practice.

The readout from antibiogram-based screening assays contains no information about the class and the therapeutic potential of the antimicrobial. Characteristics of an ideal antibiotic screening procedure should include: (i) High sensitivity and independence of bacterial susceptibility. (ii) Detection of bioavailable drugs which are taken up by most eukaryotic cells to enable treatment of intracellular pathogens. (iii) Cytotoxicity assessment of the screened compound. (iv) Simple and speedy screening set-up which avoids purification/concentration steps and facilitates detection of antibiotics with problematic stability. (v) Adaptation to robotic high-throughput screening technology. Specificity for a small class of compounds having proven therapeutic efficacy, such as streptogramins, 
can also be an advantage. The mammalian cell-based screening technology meets favorably with all requirements for an ideal antibiotic detection system.

The mammalian cell-based screening system is particularly sensitive and detects the model streptogramin pristinamycin at nanogram concentrations $(50 \sim 100 \mathrm{ng} / \mathrm{ml})$ in metabolic libraries. Thus, it outperforms antibiogram tests using the most streptogramin-sensitive $C$. diphtheriae as indicator bacteria by a factor of at least 20 . The minimal inhibitory concentration (MIC) which defines the sensitivity of antibiogram tests is $2 \mu \mathrm{g} / \mathrm{ml}$ for streptogramins (SFM; published by Sanofi Diagnostics Pasteur, 3 boulevard Raymond Poincaré, 92430 Marnes la Coquette, France). The superior sensitivity of the cell-based screening system is surprising since the antibiogram test takes advantage of the synergistic effect which increases the bacteriocidal effect of the composite streptogramin (type I and II) by at least a factor of $100^{9,10,23)}$. Instead of the physiological readout of bacterial lysis or growth inhibition, the mammalian cell-based streptogramin detection technology uses a direct molecular interaction between the putative streptogramin antibiotic and the bacterial sensor protein Pip. The signal of the antibioticprotein interaction is linked to an engineered mammalian transcription set-up and leads to modulation of reporter gene expression following addition of a streptogramincontaining culture supernatant or even a single microbial colony $^{21 \sim 23)}$. Our results indicate that the PipON and PipOFF systems, which have originally been designed as human-compatible, pristinamycin-responsive gene regulation concepts for regulated gene expression in mammalian cells, were responsive to all available streptogramin antibiotics ${ }^{23)}$ (Figure 1). This multipeptide sensor capacity of Pip is the basis for the streptogramin screening technology.

Multidrug recognition of proteins modulating expression of antibiotic resistance determinants has previously been described for the Streptomyces thiostrepton-induced protein TipAL and the B. subtilis multidrug resistance regulatory protein $\mathrm{BmrR}^{40,41)}$. Two structurally different substrates of the B. subtilis multidrug transporter Bmr, rhodamine $6 \mathrm{G}$ and tetraphenylphosphonium, activate transcription of $\mathrm{bmr}$ in a positive feedback regulation circuit by interaction with $\mathrm{BmrR}^{40)}$. TipAL, which shows homology to $\mathrm{BmrR}$, is a multipeptide sensor specific for cyclic thiopeptide antibiotics having dehydroalanine side chains ("tails"). Following covalent interaction of thiopeptide antibiotics such as thiostrepton, nosiheptide, berniamycin and promothiocin, TipAL autogenously activates its own transcription by binding to the tipA promoter $\left(\mathrm{P}_{\text {tipA }}\right)^{41)}$. The potential of TipAL to interact with thiopeptides of heterogeneous primary structure was used to design a screening tool for the detection of novel thiopeptide antibiotics. When antibiogram discs loaded with actinomycete metabolite libraries were placed onto a lawn of Streptomyces lividans harboring a $\mathrm{P}_{\text {tipA }}$-driven kanamycin resistance construct, induction of $\mathrm{P}_{\text {tipA }}$ could be visualized by a zone of kanamycin-resistant growth whose diameter was dependent on both the kanamycin concentration in the plate and the amount of thiopeptide inducer in the antibiogram disc ${ }^{42)}$. This $\mathrm{P}_{\text {tipA }}$-induction test was successfully used for the discovery of 15 new compounds which had quite different chemical structures but were all thiopeptides having antibiotic activity ${ }^{41,43 \sim 46)}$.

Bacteria are inherently sensitive to antibiotics and some bacterial promoters respond nonspecifically to antibiotic-induced stress. For example, the antibiotics chloramphenicol, spiramycin, streptomycin, tetracycline, rifamycin, and oleandomycin were able to induce kanamycin resistance in $S$. lividans harboring Pptr-driven kanamycin cassettes ${ }^{20)}$. Unlike pristinamycin, these antibiotics did not release Pip from Pptr in gel retardation assays ${ }^{23)}$, nor modulate $\mathrm{P}_{\mathrm{PIR}}$ or $\mathrm{P}_{\mathrm{PIR}} \mathrm{ON}$-driven SEAP expression in mammalian cells at concentrations of $2 \mu \mathrm{g} / \mathrm{ml}$ (Figure 1 and Figure 2).

Streptogramins are a unique class of antibiotics which show a broad spectrum of activity against multidrugresistant Gram-positive and a variety of Gram-negative human pathogenic bacteria and display a very low potential to elicit antibiotic resistance ${ }^{2,9)}$. The semisynthetic pristinamycin derivative Synercid ${ }^{\circledR}$, composed of dalfopristin, a 26-sulphonyl derivative of PII, and quinupristin, which is derived from PI by synthetic addition of a $(5 \delta R)$-[(3S)-quinuclidinyl] thiomethyl group has recently attracted much interest as a last line of defence against untreatable multidrug-resistant pathogens ${ }^{5,9,11)}$. Despite the potential of streptogramins for human therapy, little attention has been focused on the discovery of novel streptogramin antibiotics in the past decades. The mammalian cell-based screening technology presented here has the potential to detect novel streptogramin activities for application in human therapy. Although the use of the PipON and PipOFF systems is limited to the detection of streptogramin antibiotics, this technology may be applied to screening modalities for other classes of antibiotics using regulatory proteins found in a variety of antibiotic producing organisms or resistant pathogens ${ }^{47 \sim 51)}$. Similar adaptation of these resistance regulons for use as antibiotic detection systems will likely become a powerful tool in drug discovery, to restrain the increasing prevalence of 
MDR human pathogenic bacteria.

\section{Acknowledgements}

We thank E. Niederer for FACS sorting, P. VIOLLIER and F. RENAUD for their generous advice during the project. This work was supported by Cistronics Cell Technology $\mathrm{GmbH}$, Einsteinstrasse, P.O. Box 145, CH-8093 Zurich, Switzerland.

\section{References}

1) Nagaraja, T. G. \& M. M. Chengappa: Liver abscesses in feedlot cattle: a review. J. Anim. Sci. 76: 287 298, 1998

2) BAQUero, F.: Gram-positive resistance: challenge for the development of new antibiotics. J. Antimicrob. Chemother. 39: 1 6, 1997

3) Davies, J.: Inactivation of antibiotics and the dissemination of resistance genes. Science 264: 375 382,1994

4) Gold, H. S. \& R. C. Moellering Jr.: Antimicrobialdrug resistance. N. Engl. J. Med. 335: 1445 1453, 1996

5) LeClercQ, R. \& P. Courvalin: Streptogramins: an answer to antibiotic resistance in gram-positive bacteria. Lancet 352: 591 592, 1998

6) LiU, H. H.: Antibiotic resistance in bacteria. A current and future problem. Adv. Exp. Med. Biol. 455: 387 396, 1999

7) Neu, H.: The crisis in antibiotic resistance. Science 257: 1064 1072, 1992

8) Rosamond, J. \& A. Allsop: Harnessing the power of the genome in the search for new antibiotics. Science 287 : $1973 \sim 1976,2000$

9) Barrière, J. C.; D. H. Bouanchaud, J. F. Desnottes \& J. M. PARIS: Streptogramin analogues. Expert Opin. Invest. Drugs 3: 115 131, 1994

10) Cocito, C.; M. Di Giambattista, E. Nyssen \& P. VANNUFFEL: Inhibition of protein synthesis by streptogramins and related antibiotics. J. Antimicrob. Chemother. 39: 7 13, 1997

11) Barrière, J. C.; N. Berthaud, D. Beyer, S. DutkaMalen, J. M. Paris \& J. F. Desnottes: Recent developments in streptogramin research. Curr. Pharm. Des. 4: $155 \sim 180,1998$

12) Cocito, C.: Antibiotics of the virginiamycin family, inhibitors which contain synergistic components. Microbiol. Rev. 43: 145 198, 1979

13) BÉBÉAR, C. \& D. H. BOUANCHAUD: A review of the invitro activity of quinupristin/dalfopristin against intracellular pathogens and mycoplasmas. J. Antimicrob. Chemother. 39: 59 62, 1997

14) Bergeron, M. \& G. Montay: The pharmacokinetics of quinupristin/dalfopristin in laboratory animals and humans. J. Antimicrob. Chemother. 39: 129 138, 1997

15) Pechere, J. C.: Streptogramins. A unique class of antibiotics. Drugs 51: 13 19, 1996

16) Allignet, J.; N. Liassine \& N El SOlH: Characterization of a staphylococcal plasmid related to pUB110 and carrying two novel genes, vatC and $\mathrm{vgbB}$, encoding resistance to streptogramins $A$ and $B$ and similar antibiotics. Antimicrob. Chemother. 42: 1794 1798,
1998

17) Lina, G.; A. Quaglia, M. E. Reverdy, R. LeclerQ, F. VANDEnESCH \& J. Etienne: Distribution of genes encoding resistance to macrolides, lincosamides, and streptogramins among staphylococci. Antimicrob. Agents Chemother. 43: 1062 1066, 1999

18) Blanc, V.; K. Salah-Bey, M. Folcher \& C. J. THOMPSON: Molecular characterization and transcriptional analysis of a multidrug resistance gene cloned from the pristinamycin-producing organism, Streptomyces pritsinaespiralis. Mol. Microbiol. 17: $989 \sim 999,1995$

19) LeE, C.; Y. Kamitani, T. Nihira \& Y. Yamada: Identification and in vivo functional analysis of a virginiamycin $S$ resistance gene (varS) from Streptomyces virginiae. J. Bacteriol. 181: 3293 3297, 1999

20) Salah-Bey, K.; V. Blanc \& C. J. Thompson: Stressactivated expression of a Streptomyces pristinaespiralis multidrug resistance gene (ptr) in various Streptomyces spp. and Escherichia coli. Mol. Microbiol. 17: 1001 1012,1995

21) SALAh-Bey, K. \& C. J. Thompson: Unusual regulatory mechanism for a Streptomyces multidrug resistance gene, ptr, involving three homologous protein-binding sites overlapping the promoter region. Mol. Microbiol. 17: $1109 \sim 1119,1995$

22) Folcher, M.; R. P. Morris, G. Dale, K. Salah-BeyHocrni, P. Viollier \& C. J. ThOMPSON: A transcriptional regulator of a pristinamycin resistance gene in Streptomyces coelicolor. J. Biol. Chem. 276, 2001

23) Fussenegger, M.; R. P. Morris, C. Fux, M. Rimann, B. von Stockar, C. J. Thompson \& J. E. Bailey: Novel streptogramin-based gene regulation systems for mammalian cells. Nat. Biotechnol. 18: 1203 1208, 2000

24) KHOKOLOV, A. S.: Low molecular weight microbial bioregulators of secondary metabolism. In Overproduction of Microbial Products, Eds. V. KRUMPHANZEL, B. SikytA, Z. \& VANEK, pp. 97 109, Academic Press, London, 1982

25) Retzlaff, L.; G. Mayer, S. Beyer, J. Ahert, S. Verseck, J. Distler \& W. Piepersberg: Streptomycin production in Streptomyces: A progress report. In Industrial Microorganisms: Basic and applied molecular genetics, Eds. R. H. BAltz, G. D. Hegeman, \& P. L. SKATRUD, pp. 183 194., American Society for Microbiology, Washington D. C., 1993

26) Fussenegger, M.; S. Schlatter, D. DÄtwyler, X. MAZUR \& J. E. BAILEY: Controlled proliferation by multigene metabolic engineering enhances the productivity of Chinese hamster ovary cells. Nat. Biotechnol. 16: 468 472, 1998

27) Berger, J.; J. Hauber, R. Hauber, R. Geiger \& B. R. CULLEN: Secreted alkaline phosphatase: a powerful new quantitative indicator of gene expression in eukaryotic cells. Gene 66: 1 10, 1988

28) Bronstein, I.; J. Fortin, P. E. Stanley, G. S. Stewart \& L. J. KRICKA: Chemiluminescent and bioluminescent reporter gene assays. Anal. Biochem. 219: 169 181, 1994

29) LEVy, S. B.: Multidrug resistance -a sign of times. N. Engl. J. Med. 338: 1376 1378, 1998 
30) De Boer, O. J.; A. C. van Der Wal \& A. E. Becker: Atherosclerosis, inflammation, and infection. J. Pathol. 190: $237 \sim 243,2000$

31) Meier, C. R.; L. E. Derby, S. S. Jick, C. Vasilakis \& H. JICK: Antibiotics and risk of subsequent first-time acute myocardial infarction. JAMA 281: 427 431, 1999

32) Levenstein, S.: Peptic ulcer at the end of the 20th century: biological and psychological risk factors. Can. J. Gastroenterol. 13: 753 759, 1999

33) Goldenberg, D. L: Bacterial arthritis. Curr. Opin Rheumatol. 7: 310 314, 1995

34) Keat, A.: Reactive arthritis. Adv. Exp. Med. Biol. 455: 201 206, 1999

35) Demain, A. L. \& R. P. Elander: The beta-lactam antibiotics: past, present, and future. Antonie Van Leeuwenhoek 75: 5 19, 1999

36) Hamilton-Miller, J. M.: Beta-lactams: variations on a chemical theme, with some surprising biological results. J. Antimicrob. Chemother. 44: 729 734, 1999

37) MATA, E. G.: Solid-phase and combinatorial synthesis in beta-lactam chemistry. Curr. Pharm. Des. 5: 955 964, 1999

38) Alvarez, M. A.; H. Fu, C. Khosla, D. A. Hopwood \& J. E. BAILEY: Engineered biosynthesis of novel polyketides: properties of the whiE aromatase/cyclase. Nat. Biotechnol. 14: 335 338, 1996

39) Xue, Q.; G. Ashley, C. R. Hutchinson \& D. V. Santi: A multiplasmid approach to preparing large libraries of polyketides. Proc. Natl. Acad. USA 96: 11740 11745, 1999

40) Ahmed, M.; C. M. Borsch, S. S. Taylor, N. VazQuezLASLOP \& A. A. NEYFAKH: A protein that activates expression of a multidrug efflux transporter upon binding the transporter substrates. J. Biol. Chem. 269: 28506 285013, 1994

41) Chiu, M. L.; M. Folcher, T. Katoh, A. M. Puglia, J. Vohradsky, B.-S. Yun, H. Seto \& C. J. Thompson: Broad spectrum thiopeptided recognition specificity of the Streptomyces lividans TipAL protein and its role in regulating gene expression. J. Biol. Chem. 274: 20578 20586, 1999

42) Murakami, T.; T. G. Holt \& C. J. Thompson: Thiostrepton-induced gene expression in Streptomyces lividans. J. Bacteriol. 171: 1459 1466, 1989
43) Yun, B. S.; T. Hidaka, K. Furihata \& H. Seto: Microbial metabolites with tipA promoter inducing activity. III. Thioxamycin and its novel derivative, thioactin, two thiopeptides produced by Streptomyces sp. DP94. J. Antibiotics 47: 1541 1545, 1994

44) Yun, B. S. \& H. SETO: Promoinducin, a novel thiopeptide produced by Streptomyces sp. SF2741. Biosci. Biotechnol. Biochem. 59: 876 880, 1995

45) Yun, B. S.; T. Hidaka, K. Furihata \& H. Seto: Microbial metabolites with tipA promoter inducing activity. II. Geninthiocin, a novel thiopeptide produced by Streptomyces sp. DD84. J. Antibiotics 47: 969 975, 1995

46) Yun, B. S.; T. Hidaka, K. Furihata \& H. Seto: Promothiocins $\mathrm{A}$ and $\mathrm{B}$ novel thiopeptides with a tipA promoter inducing activity produced by Streptomyces $\mathrm{sp}$. SF2741ics J. Antibiotics 47: 510 514, 1994

47) August, P. R.; L. TANG, Y. J. YoON, S. Ning, R. MüLleR, T.-W. M. Yu, Taylor, D. Hoffmann, C.-G. Kim, C. R. Hutchinson \& H. G. Floss: Biosynthesis of the ansamycin antibiotic rifamycin: deductions from the molecular analysis of the rif biosynthetic gene cluster of Amycolatopsis mediterranei S699. Chem. Biol. 5: 69 79, 1998

48) Guilfoile, P. G. \& C. R. Hutchinson: The Streptomyces glaucescens $\mathrm{TcmR}$ protein represses transcription of the divergently oriented $t c m R$ and $t c m A$ genes by binding to an intergenic operator region. J. Bacteriol. 174: 3659 3666, 1992

49) Martin, J. F. \& P. LiRas: Organization and expression of genes involved in the biosynthesis of antibiotics and other secondary metabolites. Annu. Rev. Microbiol. 43: 173 206, 1989

50) Poirel, L.; M. Guibert, D. Girlich, T. NaAs \& T. NORDMANN: Cloning, sequence analyses, expression and distribution of ampC-ampR from Morganella morganii clinical isolates. Antimicrob. Chemother. 43: 769 776, 1999

51) Schwecke, T.; J. F. Aparicio, I. Molnar, A. König, L. E. KhaW, S. F. HAYDOCK, M. OliyniYK, P. CAFFrey, J. Cortes, J. B. Lester, G. A. Böhm, J. O. Staunton \& P. F. LEADLAY: The biosynthetic gene cluster for the plyketide immunosuppressant rapamycin. Proc. Natl. Acad. Sci. USA 92: 7839 7843, 1995 